\title{
Mediaciones en el universo de las policlínicas de atención primaria. Un ejercicio etnográfico sobre los flujos de comunicación e información en el campo de la salud.
}

\author{
Eduardo Álvarez Pedrosian' \\ Recebido em: 12/03/2013. Aprovado em: 25/04/2013. Disponibilizado em: 26/06/2013
}

1. Pos-Doctorando en Antropología por la Universidad de São Paulo (USP, Brasil), Doctor (Ph. D.) en Filosofía: Historia de la Subjetividad, y Diplomado en Estudios Avanzados (DEA) en Filosofía, ambos por la Universidad de Barcelona (UB, Cataluña, España), y Licenciado en Ciencias Antropológicas por la Universidad de la República (UdelaR, Uruguay). En esta última se desempeña como Profesor Adjunto en Régimen de Dedicación Total (RDT) del Departamento de Ciencias Humanas y Sociales, Licenciatura en Ciencias de la Comunicación e investigador del Programa de Desarrollo Académico de la Información y la Comunicación (DCHS-LICCOM-PRODIC-UdelaR). Miembro del Sistema Nacional de Investigadores (SNI-ANII, Uruguay).

\section{Resumo}

Mediações no universo das policlínicas de atenção primária. Um exercício etno-gráfico sobre os fluxos de comunicação e informação no campo da saúde. Neste artigo analisamos os fluxos de comunicação e informação em uma sala de espera de uma poli-clínica média a nordeste da região metropolitana de Montevidéu (Uruguai). Realizamos uma prática etnográfica num contexto de instituições inter-relacionadas, na policlínica localizada em COVIPRO, uma pequena localidade em uma rota afetada por fortes mu-danças territoriais. Estudamos os tipos de mediadores do sistema de saúde, as estraté-gias de informação e comunicação, e a geração de territórios informacionais em distintas mediações sanitárias, especialmente no espaço-tempo de espera. Finalmente, analisamos algumas características particulares do estilo da comunicação adolescente nessas circunstâncias.

Palavras-chave: etnografia, mediações, comunicação, informação, atenção primária e saúde pública.

\begin{abstract}
In this article analyzes the flow of communication and information on a waiting hall of a middle polyclinic situated in a Northwest Zone of Montevideo Department (Uruguay). In the context of an interwoven of institutions, we made the ethnographic practice in the polyclinic located in COVIPRO, small locality alongside a road affected by strong territorial changes. We studied the types of health mediators and their communication and information strategies and the generation of informational territories, in different sanitary mediations, specially in the waiting space-time. Finally, are described some characteristics of adolescent style of communication in these circumstances.
\end{abstract}

Keywords: ethnography, mediations, communication, information, primary care and public health. 


\section{Resumen}

Mediations at the universe of the primary care polyclinics. An ethnographic ex-ercise about the flow of communication and information on the field of health. En este articulo se analizan los flujos de comunicacion $e$ información en una sala de espera de una policlinica media en la zona nordeste del Departamento de Montevideo (Uruguay). En el contexto de un entretejido de instituciones, se llevó a cabo la práctica etnográfica en la policlínica ubicada en COVIPRO, pequeña localidad sobre una ruta afectada por fuertes cambios territoriales. Se estudian los tipos de mediadores del sistema sanitario y sus estrategias de información y comunicación y la generación de territorios informa-cionales en las distintas mediaciones sanitarias, especialmente en el espacio-tiempo de la espera. Por último, se señalan algunas características particulares del estilo de comu-nicación adolescente en tales circunstancias.

Keywords: etnografía, mediaciones, comunicación, información, atención primaria y salud pública.

PEDROSIAN,E.A.: Mediaciones en el universo de las policlínicas de atención primaria. Un 


\section{Introducción}

Esta investigación se inscribe en un proyecto más extenso, denominado "Las estrategias de información y comunicación centradas en los jóvenes y adolescentes en el ámbito de la salud: análisis y propuestas (Red de Atención Primaria (RAP) - Administración de los Servicios de Salud del Estado (ASSE), Zona 9 de Montevideo) ${ }^{1 "}$. Una de nuestras tareas particulares en el equipo interdisciplinario, consistió en realizar este acercamiento etnográfico a la realidad de las policlínicas de atención de primer nivel, en el entendido de que es en la cotidianidad de tales espacio-tiempos donde los procesos comunicacionales e informacionales estudiados se territorializan de forma, hasta ahora, más intensa y decisiva.

La Zona 9 del Departamento de Montevideo considerada por la institución esta-tal de salud, corresponde en gran medida a lo que cultural y geográficamente constituye una larga franja lineal ubicada a lo largo de un antiguo camino (hoy avenida dentro de la ciudad de Montevideo, luego camino sub-urbano, y posteriormente ruta nacional). Va-riados territorios locales se yuxtaponen, solapan y aglomeran a lo largo de la ruta, junto a campos abiertos, algunas pequeñas zonas de huertas y chacras rurales, extendiéndose más allá del límite departamental hacia otras ciudades importantes. Las poblaciones que habitan allí muestran altos niveles de exclusión y precariedad social, siendo particular-mente evidente en jóvenes y adolescentes.

\footnotetext{
${ }^{1}$ Proyecto de investigación coordinado por Martha Sabelli (Depto. de Información y Sociedad, EUBCA), Jorge Rasner (Área de Teoría de la Comunicación, LICCOM) y Raúl Ruggia (Instituto de Computación, FING), y financiado por el Programa de Desarrollo Académico de la Información y la Comunicación (PRODIC-CSIC-UdelaR), Uruguay, 2010-2012.
}

En este contexto se investigan los fenómenos comunicacionales y el manejo de la información en su articulación con los procesos de subjetivación en la conformación de "estilos de vida" (Savolainen, 2008), las configuraciones existenciales generadas desde prácticas concretas, definiendo estéticas singulares (Guattari, 1996). Ello implica, en primer término, pasar del estudio de "los medios a las mediaciones": "que el eje del debate se desplace... a las articulaciones entre prácticas de comunicación y movimientos sociales, a las diferentes temporalidades y la pluralidad de matrices culturales" (MartínBarbero, 2003: 257).

Entre estas últimas se ubican las valorizaciones y sentidos otorgados a la salud, enfermedad, dolor, vida y muerte. Por ello la OMS asume la necesidad de comprender la "calidad de vida" en tanto "percepción de los individuos de su posición en la vida en el contexto de la cultura y del sistema valórico en el que viven y en relación con sus metas, expectativas, normas e intereses” (Lolas Stepke, 1997: 160).

La inmanencia de este campo de relaciones humanas, se traduce en procesos de comunicación donde el denominado caraa-cara sigue siendo el medio por excelencia, así como se pone en juego la espacialidad y temporalidad de los lugares, los cuales son mu-cho más que escenarios neutros de los fenómenos estudiados. Las policlínicas se distribuyen de forma diferencial por toda la zona, en una red institucional compleja donde varía el tipo de atención sanitaria brindada, la población que efectivamente hace uso de los servicios, tanto en sus cualidades como en su cantidad. Algunas son fruto de la labor de colectivos vecinales organizados, quienes logran administrar un local que oficia de instalación edilicia, mientras las autoridades estatales $O$ municipales se encargan del funcionamiento brindando la atención, otras lo son a través 
de alguna institución religiosa, que aporta el espacio y la energía eléctrica, etc. En el mosaico de entornos veci-nales característico de la periferia montevideana, y siguiendo la lógica lineal de disposi-ción territorial de la zona estudiada, las policlínicas poseen diferentes grados de inmer-sión y alcance, por la atención brindada, las instalaciones específicas para ello y la accesibilidad de la población.

\section{Un entretejido de instituciones}

Tomando en cuenta este entretejido de instituciones localizadas que se superpone al mosaico de los bordes de la ciudad desdibujada, hemos identificado tres tipos de policlínicas, tres tipos de entornos de relacionamiento que nos permiten caracterizar diferencialmente la forma en que se suscitan los fenómenos comunicacionales y en ellos el tipo de manejo de la información. Estos tipos pueden comprenderse como dimensio-nes cualitativamente diferentes que no dejan de tener correspondencias cuantitativas en aspectos, como ser, la población efectivamente asistida, el número de territorios de pro-cedencia de los integrantes de la misma, la variedad de especialidades y servicios ofre-cidos en sus instalaciones, etc. Haciendo un cambio de escalas por los ajustes con el uni-verso de estudio abordado en Del Castillo, et. al. (2012), y estableciendo la escala micro por encima de la vivienda y hogar, en el ámbito territorial local, consideramos a la di-mensión meso o media como la relativa a sub-zonas o microregiones existentes, y como macro a la zona lineal en su conjunto, dispuesta a ambos lados del Camino Maldonado-Ruta 8.

En una escala micro, existen policlínicas como la de El Monarca, donde sus usuarios se caracterizan por ser del propio territorio local, el asentamiento devenido en barrio, y de los otros territorios contiguos, como el asentamiento La Rinconada (rincón literal del Departamento de Montevideo hacia el nordeste), Villa Isabel o Paso Hondo.

En otra dimensión de tipo intermedia o meso, nos encontramos con policlínicas como la de COVIPRO, donde el uso realizado de la misma conlleva la extensión hasta territorios más allá de los contiguos de la localidad donde se encuentra instalada.

Cubriendo mayores distancias que las pequeñas policlínicas locales, pero sin de-jar de ser interna a la zona considerada, este tipo de espacios de interacción combinan las dinámicas típicamente próximas con las que denotan otras distancias sociales, desconocimientos y anonimatos (Simmel, 2005) casi imposibles de encontrar en los casos anteriores.

Y por último, en un tercer nivel de organización institucional y funcionamiento concreto de la red de policlínicas de atención primaria, nos encontramos con la policlí-nica Jardines del Hipódromo, conocida en la zona por el primer término del nombre, $y$ que constituye el "centro de salud" de la misma, el foco de irradiación establecido por las autoridades sanitarias, quedando todas las demás instalaciones bajo su dependencia. Este centro muestra otro tipo de espacialidades en las que asoma la complejidad hospi-talaria (Benech y Sprechmann, 1995), aunque la falta de internación marca fuertemente la diferencia.

El tipo y cantidad de especialidades médicas, las capacidades edilicias y profesionales puestas allí en juego, la convierten en un campo de experiencias donde se conjugan usuarios que habitan en toda la zona, donde se centralizan muchas acciones, por derivación o directamente. Es importante tener presente que en cada situación localiza$\mathrm{da}$, en cualquiera de los tres niveles o escalas, 
existen cuestiones que tienden a la particularidad de lo local y otras que responden a la extensión de la globalidad tanto zonal como departamental y nacional, cuestión marcada principalmente por las actividades de los profesionales que brindan sus servicios, ya que los usuarios sí se circunscriben a sus territorios cotidianos y a lo sumo a la zona más en general.

La puesta en práctica de la observación participante conllevó un desafío por de-más relevante. Con excepción de la policlínica "Jardines" (del Hipódromo, centro de gestión de la red de atención zonal), las policlínicas de mediano y pequeño tipo consti-tuyen contextos fuertemente caracterizados por la casi exclusiva participación de muje-res, algunas junto y acompañando a hijos propios y de próximos, lo que amplía el universo de usuarios al familiar, más específicamente al de la crianza y cuidado de niños, jóvenes y adolescentes.

En algunas ocasiones lo familiar desborda en lo comunitario, los vínculos prima-rios son absorbidos en un continuum con alcance local. Pero todo ello se hace desde las mujeres, en un contexto de transformación de todas estas instituciones, roles y configu-raciones subjetivas en general. Igualmente, y quizás aún más, constituyen los agentes principales en los escenarios que conforman nuestro campo de estudio, siendo "una in-fluencia decisiva en la conformación de comportamientos relacionados con la promoción de la salud y calidad de vida" (Ludueña, Olson, Pasco, 2005: 1132), en todo caso definitorios de las disposiciones de los jóvenes y adolescentes en composición con lo que reciben desde el lado del saber médico, por lo general planteado en "otro plano de comunicación" (Peñaranda-Correa, 2011).

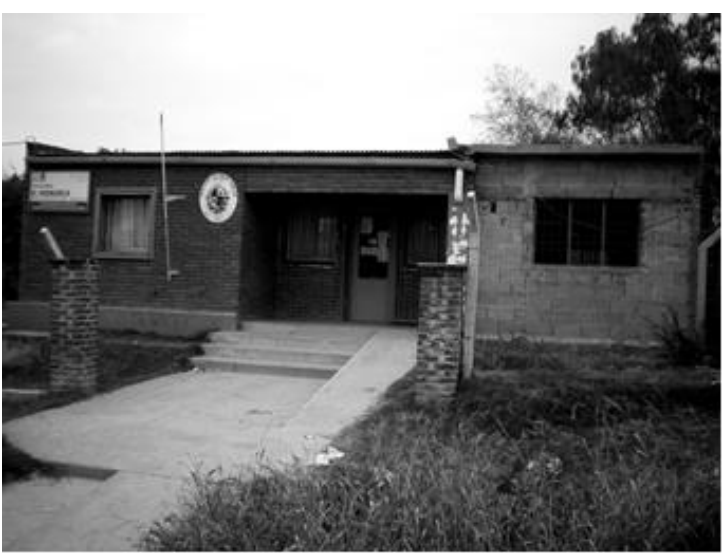

Figura 1: Policlínica E1 Monarca

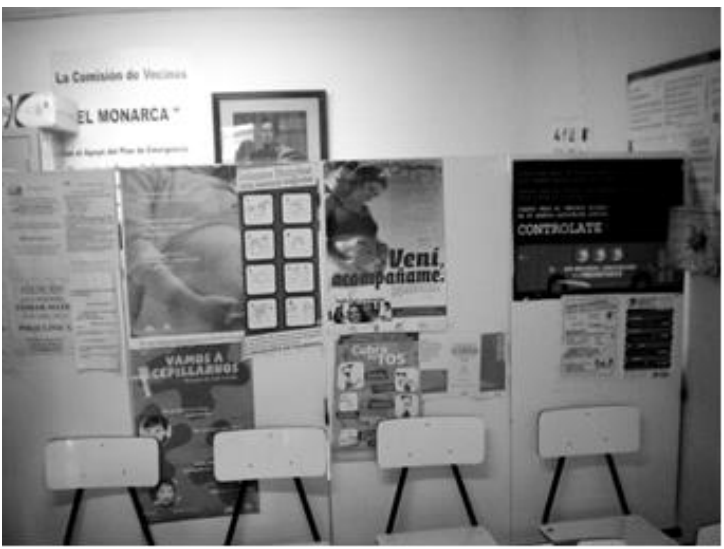

Figura 2: Sala de espera

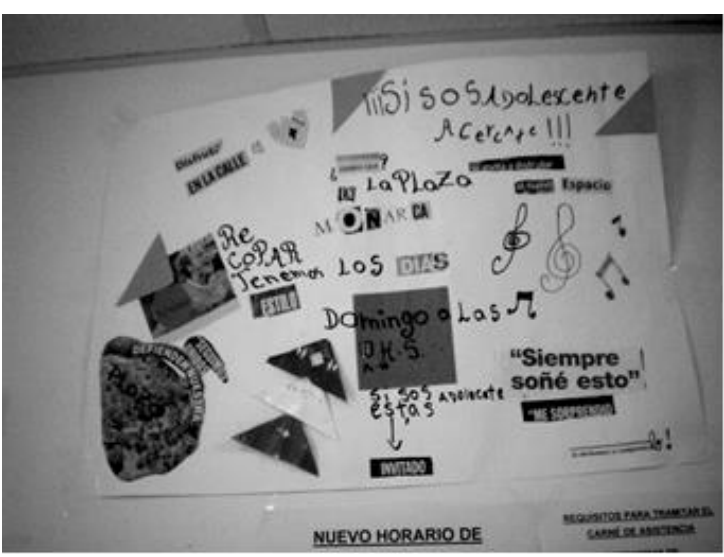

Figura 3: Cartelera orientada a jóvenes y adolescentes 


\section{A medio camino: COVIPRO entre lo barrial y lo zonal}

La zona de estudio se caracteriza por su linealidad, desarrollándose en torno a una centenaria traza territorial que va siendo avenida, camino y ruta, según la proximi-dad a la ciudad de Montevideo. A uno y otro lado de la traza, a esta altura ya ruta nacional, se organizan las diversas unidades territoriales que constituyen la zona Nordeste del Departamento de Montevideo. La ubicación relativamente intermedia de la policlínica COVIPRO, la convierte en candidata para ser referencia para los habitantes de otros territorios dispuestos a uno de los lados de la larga traza de comunicación vial.

La policlínica ha pasado de ser una más de alcance local a proyectarse sobre otras localidades. Son indicadores de ello el plan de construcción de las nuevas instalaciones para la policlínica, y principalmente el tipo de usuarios del sistema que efectivamente se encuentran cotidianamente presentes: vecinas en su mayor parte que se reconocen y saludan y reúnen por un tiempo en la sala de espera, y otras que acuden a inscribirse como usuarias nuevas, llegadas de localidades ubicadas hacia el sur hasta el llamado Kilómetro 16, o de otras en sentido norte por lo menos hasta los límites departamentales (El Monarca y La Rinconada).]

COVIPRO es la sigla de Cooperativa de Viviendas El Progreso, creada a partir de un conjunto de 50 familias, con sus hogares esparcidos sobre la cara sur de la ruta. Entre 1988 y 1993 se da un proceso temprano de regularización, apoyado por una organización no gubernamental todavía existente.

El Centro de Participación Popular (CPP), apoyó a los vecinos con formación, acompañamiento en el proceso legal y en la dinámica del colectivo en gestación (como el reglamento de convivencia) (CPP, s/d). Se obtuvo la propiedad del suelo y la legalidad plena. En la actualidad nos encontramos con una localidad ampliamente crecida, y en una ubicación que la incluye dentro de un área que recientemente ha experimentado fuertes transformaciones. El contraste es intenso y se ha acrecentado en los últimos años: por un lado la existencia de este barrio cooperativo, que siguió siendo considerado un asentamiento por las autoridades (INE, 2005), y por el otro, más próximo a la ciudad capital y ya llegando a extenderse en frente sobre la ruta, la llamada Zona América, polo de desarrollo tecnológico y de negocios transnacionales.

El hecho de que se trate de un colectivo que comenzó siendo un asentamiento irregular (cantegril en los términos tradicionales), y que se conformó como conjunto de viviendas de tipo cooperativo (con la importancia que tiene dicho movimiento social asociado a la vivienda en la sociedad uruguaya), nos hace reflexionar acerca de las par-ticularidades en las que se ubica esta policlínica que ha ido creciendo y ha saltado a una nueva escala en la atención sanitaria.

Un tercer elemento de este escenario comunicacional, es el relativo a los funcionarios del sistema sanitario que trabajan allí, pues es de anotar la existencia de una fuerte presencia de la medicina familiar y comunitaria, lo que potencia la atracción que genera dentro de la zona, dado el perfil y tipo de atención integral realizada. Resulta muy interesante que antes de nuestra entrada a este campo de experiencias (2010-2012), se haya llevado a cabo un trabajo de intervención y promoción que tuvo a la policlínica involucrada en ello. Desde un taller de radio para jóvenes y adolescentes generado en uno de los liceos de la zona, en 
la conjunción de la psicología, las ciencias de la educa-ción y las de la comunicación, surgió una experiencia que involucró a la radio comuni-taria En Libertad 106.3 FM, desde donde unos sesenta participantes elaboraron conteni-dos sobre salud. (Cabrera, Ricardo, Bresciano, 2009).

Una circunstancia, que nos pone de manifiesto lo intempestivo y ligado al acontecimiento que se encuentra el conocimiento en ciencias humanas y sociales, y por ello mismo inmerso en los fenómenos que estudia, era un emergente de este proceso: otra de las policlínicas de nuestra zona, la llamada 8 de Marzo, se encontraba "cerrada" según las palabras de usuarias y profesionales, con lo cual el flujo de nuevos pacientes y la expansión de los territorios cercanos de procedencia se había visto potenciado. Nos en-contramos, por tanto, con la policlínica en estado de crecimiento, consolidando su di-mensión intermedia dentro de la zona Nordeste. Servicios como el odontológico se ha-bían trasladado directamente hacia allí, y para ello se había instalado un consultorio rodante de tiro hacia un lado de un amplio terreno que sirve de interface entre la policlí-nica y la ruta, donde se encuentra su puerta.

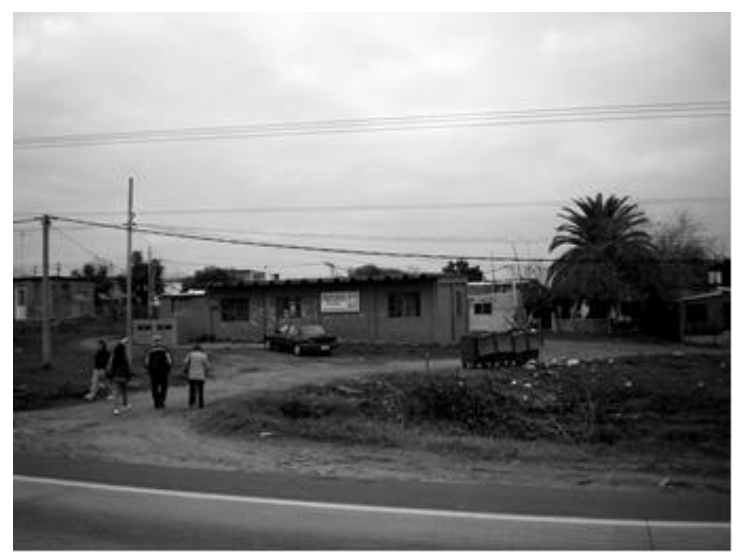

Figura 4: Policlínica COVIPRO desde la ruta

\section{Los mediadores y sus estrategias}

En nuestra primera visita a la policlínica COVIPRO nos encontramos con parte de su cuerpo médico, de enfermería y auxiliar. Entre ellos existen cruces permanentes en otras policlínicas de la zona, más allá de la misma, y en otros ámbitos del campo de la salud, incluido el universitario. En el rol de la gestión local, los llamados promotores de salud y otros perfiles igual de vecinales aunque sea de otras localidades del Departamento, son quienes ofician de mediadores principales entre el sistema y los usuarios, la institución sanitaria y las mallas locales de contextualización. En nuestro caso, una de ellas nos permitió acceder a una metodología sui generis que había elaborado para gestionar la información. Todo un protocolo, un tipo de manejo de los datos, una lógica de ordenamiento, almacenamiento y recuperación, llevada a cabo a partir de fichas escritas a mano, dentro de carpetas, dentro a su vez de un armario, constituían una estrategia de información y comunicación.

En un universo previo a la informatización, basado en las mediaciones clásicas de la escrita y lectura de textos, las fichas por paciente elaboradas son un material riquísimo para el conocimiento y saber médico, y pone en evidencia la necesidad de contar con este tipo de productos cognoscentes, sistematizaciones y organización de la información y los conocimientos para poder dar cuenta de la atención de los sujetos que así lo requieren. Esto nos permite reflexionar sobre la existencia de cierto tipo de configu-ración cotidianamente elaborada y estandarizada por el uso cuando se dan ciertas regularidades en el funcionamiento del lugar, posibles transmisiones de esos modelos $y$ dis-posiciones y sus permanentes transformaciones. 
Existe, por tanto, un tipo de habitus (Bourdieu, 2000) característico de cada policlínica localmente situada, disposiciones y rutinas que conllevan mediaciones cualitativamente diferenciadas; estilos de llevar adelante el funcionamiento cotidiano de una policlínica en un momento determinado. Estos estilos conllevan ontologías, en el sentido informático de constituir el magma subjetivo del que se desprenden los materiales a ser considerados como información, así como la lógica que los compone. En este nivel intrínseco de comunicación organizacional de la policlínica, descubrimos la generación de formas clasificatorias y compositivas en general que intentan hacer el mejor uso racional de una multiplicidad caótica y desbordante de información sobre cada paciente, familia, comunidad. En este nivel meso o medio de policlínicas, pueden encontrarse ordenadores y otros insumos tecnológicos, pero todavía existen estos casos, extremos en tal sentido, donde la escritura a mano alzada sobre fichas sigue siendo el soporte y la materia de expresión reinante.

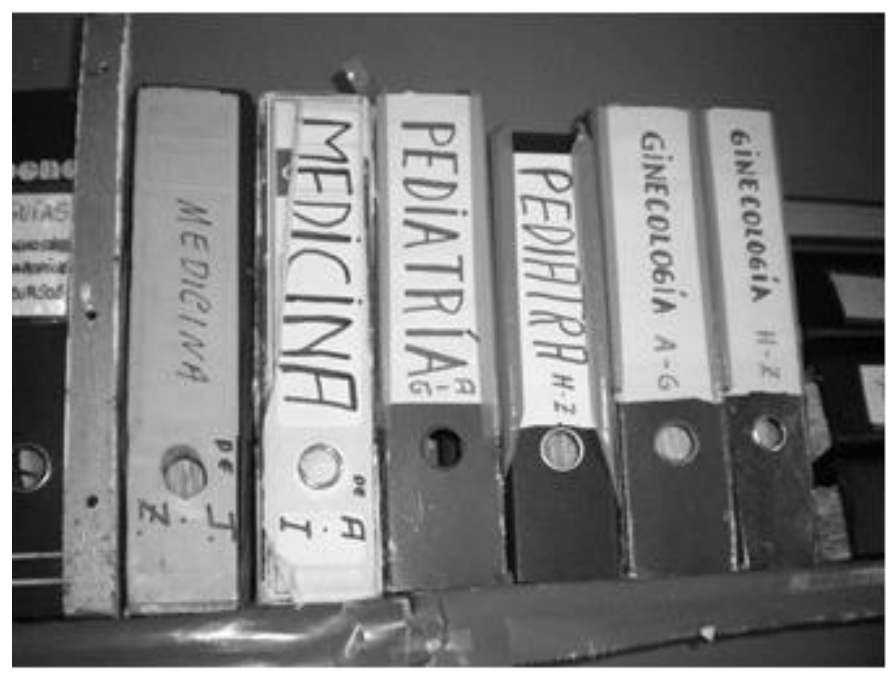

El pequeño equipo se organiza en dos turnos, siendo el mediodía quien marca el cambio. Las consultas se realizan en variados días, y optamos realizar las observaciones participantes en aquellos momentos en que, gracias a las consultas de medicina general, pediatría, ginecología, psicología y asistencia social, y según lo contrastado en entrevis-tas con algunos de los profesionales de las respectivas áreas y disciplinas, se concentraba la participación de jóvenes y adolescentes, foco de interés de la investigación en la que se inscribe este estudio. Ingresamos a un ámbito muy íntimo y femenino, tratando de adaptarnos lo mejor posible a la situación para establecer una perspectiva no intrusiva, todo un desafío. En una sala de unos $20 \mathrm{mt} 2$, se desarrolla una interminable sucesión de acontecimientos pautados por interacciones cara-a-cara, la espera para ser atendido en alguno de los servicios de salud, y es allí donde se define la eficacia o no de todo el sistema: el plano de inmanencia del contexto de experiencia.

Figuras 5 y 6: archivos sanitarios en COVIPRO 
Territorios informacionales en las mediaciones sanitarias

Como una suerte de vecino que se encuentra a la espera de alguien, llevando a cabo una constante inscripción de lo sucedido, nos ubicamos desde la primera vez en el borde de uno de los dos bancos de madera al fondo, justo donde se tocan, en medio del salón. Consideramos que en un ámbito de interacción tan pequeño y de máximas pro-ximidades, lo mejor es no pretender encontrar algún tipo de invisibilidad, porque jamás la habrá. También el paso del tiempo, durante todo un turno, conlleva momentos de silencios y pausas profundas, que más que vacíos son aperturas a lo que está más allá de los contornos del lugar, el afuera vasto y dinámico (tráfico de la ruta, obras y expansión del nuevo territorio empresarial, flora y fauna rural-urbana) en que se sitúa.

En uno de los ingresos, a media mañana, nos encontramos con la siguiente esce-na: Hacia la izquierda una madre con una pequeña niña de año y medio de edad, hacia el otro lado un grupo de tres adolescentes, una visiblemente embarazada. Detrás de ellas se ubica el acceso al baño público. Hacia delante, también sobre la misma pared, otro grupo de mujeres, esta vez de dos adultas, una de ellas recién llegada, también embarazada (según le contestó a alguien, de siete meses), que se reúnen a su vez con una madre, su joven hija y su pequeño bebé que se encontraban sentadas en medio, en dos de las sillas de plástico blancas dispersadas desde el fondo hasta allí.

Otra adulta habla por teléfono móvil mirando hacia afuera por una ventana hacia el lado derecho de la puerta y de espaldas a nosotros, lo que seguirá haciendo durante una hora. A esta disposición se le suman rápidamente usuarias esperando en una suerte de espacio intersticial entre la sala de espera y los pequeños consultorios hacia el lado izquierdo, donde uno opera de recepción, archivo y demás. Allí se ubica la auxiliar que organiza las atenciones, coordinando los números, ofreciendo los resultados de análisis a quien los vaya a retirar, inscribiendo a los nuevos usuarios, preparando y alcanzando un té caliente a alguno de los profesionales que trabajan en uno de los consultorios.

Este diagrama momentáneo de mediaciones, se disponía en una espacialidad es-pecífica, también dimensión comunicacional. Una cartelera se encuentra tras uno de los largos bancos longitudinales, hacia su extremo izquierdo. La misma está ocupada por algunos papeles impresos con datos referidos a los horarios de atención, condiciones para la gestión de documentos como el carné de asistencia, y la gestión del local (como una lista de vecinos identificados por sus nombres y los números de los solares donde se ubican sus viviendas que colaboró con dinero para el mantenimiento del pasto ubicado entre la policlínica y la ruta).

Otra superficie para la exhibición de material gráfico opera como tabique que separa la sala de espera con el intersticio entre la misma y los consultorios que incluyen a la recepción. Se trata de un par de poster de divulgación científica: uno hecho por es-tudiantes de medicina que trabajaron en un asentamiento de las inmediaciones y otro que informa sobre datos demográficos de COVIPRO y la necesidad de vigilar la hiper-tensión. Un tercer conjunto de objetos opera como soporte para la mediación entre la institución y los usuarios en la sala de espera, se trata de un mueble que también separa a la misma con dicho ámbito intersticial. Sobre la cara externa de una suerte de armario se encuentran afiches y papeles que informan sobre campañas más genéricas, promovidas por organismos públicos nacionales e internacionales, como 
el Día de la Mujer. Durante las jornadas compartidas allí nos encontramos con alguna usuaria leyendo alguno de los papeles engrapados y pegados con cinta adhesiva en la cartelera del fondo, con información referida a horarios de atención de las consultas principalmente.

En dichos momentos, o en aquellos de letargo, era el mismo etnógrafo quien realizaba la operación, disponiéndose a leer y apreciar las imágenes en general, como se dice, prestando (la) atención. Uno de los pósters está cargado de escritura, otro posee un gráfico sobre los pobladores.

Las mañanas en que tiene lugar la consulta ginecológica es por demás un contex-to de interacción radicalmente femenino, tanto adulto como adolescente. Nos atrevimos a realizar observaciones participantes en tales circunstancias, acercándonos, quizás, a figuras y roles imaginarios para las usuarias como ser la de un hombre a la espera de su pareja, la de un técnico y profesional cercano a alguna de las médicas que dan consulta, etc. En un turno completo pudo verse la aparición de un solo hombre, y su disposición fue por demás significativa. Entre tres adultas, cuatro adolescentes y una niña, y el et-nógrafo sentado al fondo de la sala, el espacio de la misma tenía la capacidad para in-cluir a varios usuarios más, pero él prefirió estar por varias decenas de minutos en un sitio: entre la puerta principal de la edificación y el acceso al ámbito donde se encuentra la recepción, dibujando una pequeña diagonal sobre un ángulo. Pudimos apreciar que dicha posición se encuentra especialmente caracterizada por un tipo de estar, un agenciamiento en los términos de la producción de subjetividad (Deleuze y Guattari, 1997), que podemos caracterizar como de estar-sinestar.
En el diagrama de interacciones que pueden describirse en todo ámbito de relacionamiento intersubjetivo, los sujetos que se posicionan allí lo hacen porque valoran y sienten que es la más adecuada a su situación y condición. La intuición y el deseo de entrar y salir rápidamente pueden encontrar eco o no en la situación concreta con la que se encuentre cualquier visitante, más o menos según el conocimiento previo de las dinámicas rutinarias del lugar. En este caso, el tiempo se hizo más largo de la cuenta, y de cualquier forma se mantuvo en dicho intermezzo que lo tenía, desde el punto de vista de los flujos de comunicación, ajeno en un campo de experiencias considerado como otro mundo de los posibles, especialmente el de las mujeres. Además de dialogar en varias ocasiones con la auxiliar, con quien estableció el único contacto cara-a-cara, lo otro que constituyó un medio tolerado por él fue la cartelera conformada por los afiches pegados en la cara externa del mueble al que hicimos referencia. Se trató de las pocas veces en que un usuario se dedicó ostensiblemente a leer y visualizar en general el material gráfico existente.

$\mathrm{Al}$ estar claramente discriminado por el género, el campo de experiencias muestra lo que todos los demás también implican: que las formas de participar, estar, interactuar y por tanto comunicarse -siempre produciendo y haciendo uso de información-, es una cuestión variable en intensidades, tipos y estilos. Hay sujeto cuando es posible esta singularización, lo que hace de cada cual un ente único y irrepetible; aquellas informa-ciones dentro de flujos de comunicación dispuesto en diversos procesos mediacionales son producidos y productores de los agentes que los encarnan. Se puede estar de forma hasta más que evidente, llamando la atención de todos los presentes, así como se puede estarsin-estar, intentar pasar desapercibido lo más 
posible (lo que, cuando se trata de un ámbito tan íntimo, una espacialidad culturalmente tan próxima (Hall, 1994), solo es posible en el lugar geométrico donde se está a la vez lo más próximo a la salida y lo más próximo a la recepción). Entre estos polos ideales se despliega un abanico de gradacio-nes, valores de una intensidad del flujo comunicacional que no es constante, homogéneo ni invariable, más bien todo lo contrario.

Además de lo anterior, se puede componer de otra forma la espera, pues es posi-ble entrar y salir cuando se desee, dentro de los límites de lo posible claro está. $\mathrm{Y}$ esta es otra práctica con la que nos hemos encontrado: en ciertos casos siempre presentes, algu-nos llegan ante la auxiliar, en el camino más corto, la pequeña diagonal, se retiran y vuelven cuando les corresponde el momento. En estos casos, presumimos, para nada despreciables en cantidad y por sus efectos en el campo de experiencias comunicaciona-les que estamos abordando, hay una mayor proporción de usuarios vecinos a la policlí-nica, habitantes del territorio donde esta se inserta. Pero hay que tomar en cuenta la mo-vilidad, dimensión que en sí hace variar las distancias gracias a las formas de desplaza-miento, de los primeros tipos de comunicación considerados por economistas y protosociólogos en los albores de las ciencias humanas y sociales (Mattelart y Mattelart, 1997).

El uso de motocicletas está extendido, reafirmándose algo que ya hemos venido planteando en otros estudios acerca de la necesidad de velocidad y maleabilidad de los desplazamientos exigidos por el desarrollo del Área Metropolitana de Montevideo específicamente (Álvarez Pedrosian, 2009a). En tal sentido, si nos encontramos con usuarias que resultaban residir en el Kilómetro 16, otras que llegaban de la Villa García contigua, en ómnibus y andando a pie al costado de la ruta, es de suponer que aquellos que se trasladan en motocicletas puedan venir de más lejos. Igualmente creemos que el radio de cobertura de la policlínica media se respeta a pesar de ello, que más que aumentar las distancias de procedencia, se potencia esta dinámica de entrada-y-salida permanente, para empezar la tramitación de la atención y luego llegar al momento correspondiente. Lo que sí se reafirma es el carácter sub-regional de la policlínica, el pasaje de mico a meso de su morfología, pues ya es más accesible desde aquellas distancias medias, desde los barrios $\mathrm{y}$ otros territorios próximos pero no ya necesariamente contiguos. Por último, para aquellos que no desean estar en la sala de espera aunque no cuenten con medios para trasladarse de allí, y tampoco se esté tan cerca ni con el tiempo como para que valga la pena ir y venir hasta la vivienda propia, el amplio terreno delantero, cubierto de pasto, frente a la ruta, sirve de sala de espera expandida y abierta en todas direcciones.

Junto a estas diferentes modalidades de la participación, que nos informan de los componentes de los procesos comunicacionales existentes, discurre el devenir entre quienes se instalan durante más de una hora aproximadamente a esperar dentro. Se ven completamente afectados por lo que sucede con quienes no lo hacen, y es que en los hechos, este contexto de interacciones no es unidimensional $y$ diferenciado absoluta-mente de lo que está fuera; como todo campo de experiencias, está hecho de flujos que conectan a otros campos. Esta misma condición rizomática de todo campo la hemos podido estudiar en la sala de espera del hospital más antiguo y culturalmente emblemá-tico para la sociedad uruguaya (Álvarez Pedrosian, 2009b, 2010).

En dichas circunstancias institucionalmente muchísimo más potentes (hospitali-zación, de una casi completa variedad de especialidades médicas, con 
usuarios de su entorno (Ciudad Vieja), zona céntrica de la ciudad y oeste departamental (más los po-bladores próximos del otro lado de la frontera), pudimos experimentar cómo se encon-traba atravesado por múltiples vectores de procesos comunicacionales, tanto con un universo médico, estatal y universitario, como con el barrio de inserción, las vidas y territorios de los usuarios internados allí y sus allegados, etc. Por tal razón, no es correcto pretender comprender los flujos de información y comunicación en este tipo de con-textos y situaciones, campos y agenciamientos, queriendo -más que distinguir- aislar un adentro de un afuera de manera concluyente.

Aquellos que pasan un tiempo cercano o superior a la hora, saliendo a conversar con alguien a la entrada, yendo a fumar un cigarrillo, a hablar por teléfono móvil con más intimidad, si bien se muestran más estables en su dinámica de estar allí, de territo-rialización en un sentido holístico, no dejan de fluctuar, de desterritorializarse (al ser convocados para salir, por la comunicación cara-a-cara con alguien o por la telefónica, etc.).

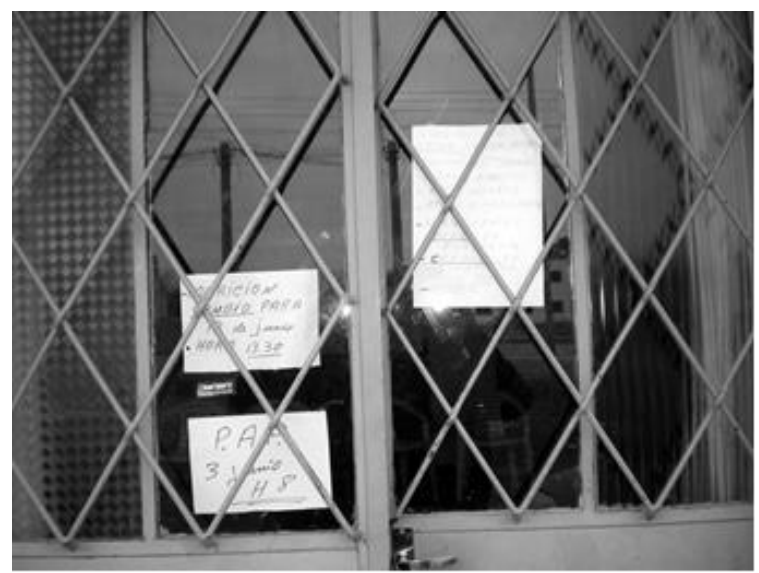

Figura 7: Puerta de Policlínica COVIPRO con notas informativas pegadas en ella

\section{E1 espacio-tiempo de la espera:} trayectos y encuentros

Este interior de la sala de espera de una policlínica media, es cualquier cosa me-nos simple y previsible (Chatman, 1999). El primer elemento central para captar su lógica y composición es comprender la dinámica de grupos que serpentea en el mismo espacio social, yendo y viniendo en el armado y desarmado de formaciones vinculares. Se trata de un espacio de encuentro principalmente entre mujeres de todas las edades, muchas de las cuales ya se encuentran relacionadas y lo seguirán estando entre sí antes y luego de reencontrarse en la sala de la policlínica.

Este tipo de aconteceres se inscriben dentro de las trayectorias de vida locales y es uno de los campos donde se juega la existencia misma del territorio: el compartir el espacio-tiempo de la sala puede significar una oportunidad de generar nuevos vínculos entre vecinas próximas o relativamente cercanas, para profundizar los ya existentes, para debilitarlos. Caldo de cultivo de las redes de reciprocidad e intercambio, cada cual con sus formas de mediación particulares, se encuentra por tanto atravesado por diversas intensidades de presencia-ausencia, tanto de usuarios como de profesionales que brindan la atención sanitaria.

Pequeños grupos de mujeres, de adultas solas, acompañadas algunas por su hija niña o adolescente, como de adolescentes solas, o ya madres, y a veces acompañadas a su vez por su madre, se conforman, se relacionan con otro y llegan a fundirse en un gru-po nuevo un poco más grande, y vuelven a desaparecer, a veces abruptamente, otras de a poco. El mate, infusión de procedencia guaraní, convertida en popular en los amplios sectores de variados estratos en una serie de sociedades de la cuenca 
sudamericana del Río de la Plata, es omnipresente.

Como se ha señalado (Vidart, 1998), el mate constituye toda una cultura así co-mo está inserto en diversas culturas regionales y opera en algunas dinámicas transcultu-rales en la actualidad. Aquí cabe recordar el carácter ritual de su ingesta, lo colectivo y propenso a generar vínculos de la misma, siendo por tanto un medio en sí mismo para el establecimiento de condiciones de comunicación e intercambio de información. Las rondas que se dibujan por los movimientos del recipiente de la bebida desde la cebadora a cada una de las que beben junto a ella, una por vez y siempre volviendo a sus manos para recargarlo de agua caliente, son expresión de los grupos, a veces de encuentros suscitados inesperadamente o muy fugaces por otras razones, y que se refuerzan por este dar y recibir, don con sabor amargo y efecto vivificante, "como una selva entera concentrada en unas gotas" (Lévi-Strauss, 1970: 156).

Estos grupos generados y regenerados a lo largo de las duraciones de los turnos de atención, de dos, tres y alguna pequeña, reunidos a su vez momentáneamente, con algunas integrantes que van y vienen porque se saludan con otra usuaria, se quedan conversando con ella o en su grupo, se expresan en mayor medida a través de aquellos medios que conforman en sus relaciones complejas la comunicación cara-acara: la ges-tualidad, la oralidad, la proxémica y la quinésica. Los sujetos, en su dimensión corporal y poniendo en juego toda su carga afectiva y libidinal en el acto, se hacen "presentes". Tenemos que comprender, si queremos analizar los procesos mediacionales, que necesi-tamos una perspectiva que vaya más allá de la distinción ausencia-presencia, y más aún en la acepción que la asocial a la dicotomía inmaterialmaterial, pues allí es donde se construye la significación, siendo el signo, justamente, una entidad imaginaria que per-mite realizar tal operación (estar sin estar, o mejor aún, estar siendo necesariamente otra cosa). Imaginario no quiere decir opuesto a lo real, por el contrario, anida en él, aunque ciertamente lo trasciende.

En un contexto considerado de lo más habitual para quienes justamente lo habi-tan, se dan acontecimientos de encuentros, generación de grupos y otras conformaciones sociológicas (mico-masas), en las que tiene cabida lo novedoso. No son tan intensamente contrastantes, como en ceremonias fuertemente codificadas, más allá del límite de lo sagrado por ejemplo, pero constituyen la red rizomática de flujos presente en toda socia-lidad, desde su grado cero en adelante. En algunas situaciones, la unidad que constituye la espacialidad de la sala se hace sentir a través de las acciones de alguna de las usuarias, preferentemente a través de la oralidad, gracias a la voz, la entonación, el discurso y sus enunciados, siempre acompañados de los demás componentes de la comunicación cara-a-cara pero trascendiéndolos por las cualidades del sonido (Ong, 1996). Un grupo de usuarias que se encuentra conversando se desmiembra cuando dos de ellas pretenden salir a fumar frente a la policlínica, una pide fuego a una adulta sola, ubicada en el centro de la parte trasera, la cual exclama con una VOz y entonación que invoca a todos los presentes (incluido el etnógrafo, a sus espaldas): "tengo fuego, soy multiuso, tengo un abanico siempre, por eso ando con cartera, cuando voy a los cursos del Ateneo tam-bién...".

Una bocanada de información densa, múltiple y compleja, sobre variados aspectos relativos a prácticas, valores y sentidos de una subjetividad en relación a otras, desde un ámbito mediano de interacción proyectándose más allá a los otros territorios de lo cotidiano. Dicha usuaria ya se encontraba visiblemente activa, emitiendo 
mensajes sin parar con sus movimientos corporales, tirando frases hacia un lado, etc. El Ateneo de Montevideo, al que hace referencia, es una institución cultural originada a fines del siglo XIX y que en la actualidad alberga actividades (cursos, conferencias, exposiciones, premiaciones) de gran amplitud de intereses, como metafísica trascendental, tango, tea-tro, declamación, divulgación científica, artes plásticas o artesanías, y se encuentra en el corazón del centro moderno de la ciudad, en el simbólico "kilómetro 0" del sistema de rutas nacionales.

Por largos momentos no hay presencia de ningún funcionario de la institución, las consultas están llevándose a cabo y discurre la espera del resto. Comparativamente a otros espacios, como el de internación hospitalaria, o consultas de tal magnitud, o de las policlínicas mayores, con sus túnicas blancas que vienen y van, a veces también en gru-po, este se muestra más articulado con el entorno local en el que se inserta, constituyen-do un tipo de territorialización institucional particularmente apropiada por los vecinos y otros usuarios cercanos. Ciertamente el carácter local del predio y la construcción, el mantenimiento de algunos servicios (como la poda del césped que media con la ruta, y no sabemos cuántos más) es expresión de ello. La nueva construcción que se realizará quizás modifique tal situación, habilitando nuevas oportunidades pero también enfrentándonos a nuevos desafíos. Dichos contextos de nula o bajísima presencia de funciona-rios de cualquier orden en la sala, son rotos por la voz de la auxiliar, parte de la conver-sación de alguna médica con su paciente dentro de alguno de los consultores contiguos, el llamado de un nuevo turno, etc.

El contexto vuelve a manifestar su matriz institucional cuando lo que acontece en el espacio intersticial contiguo invade a la sala de espera, con sus sonidos y a través del campo visual existente, interrumpido como vimos por los tamices materializados en las superficies que ofician de carteleras. La auxiliar, que tiene su archivo producido con tanto esmero en el consultorio que opera de recepción, es por excelencia lo que se denomina mediadora. Se saluda con todos los usuarios, de todas las edades, bromeando con los niños, mimoseando con los más pequeños. Por lo general se encuentra instalada allí, en dicho consultoriorecepción, y por ello el espacio que media entre el mismo y la sala de espera se convierte por momentos en el micro-lugar de mayor densidad de flujos comunicacionales, desbordándose y afectando a la totalidad de quienes se encuentran bajo el mismo techo. Y es que el límite entre la sala propiamente dicha (donde se en-cuentran bancos, sillas, la puerta principal, acceso a los baños público y para funciona-rios, y los dos consultorios hacia uno de los lados) y este pequeño entorno rectangular ubicado hacia un lado, se da a nivel superficial, con tamices verticales que afectan prin-cipalmente la visión, pero muy delgados y sin alcanzar el techo.

Toda una mediología del sonido se despliega en tal sentido, identificándose conversaciones de variada índole, siempre con la auxiliar como una de las participantes en tales encuentros. Cuando pudimos ubicarnos desde una posición que permite proyectar una mirada, y por los vacíos que se cuelan entre las superficies usadas como carteleras, guiados por la cantidad de voces, informaciones proferidas de un lado y del otro de la asistencia, nos encontramos con una pequeña aglomeración de usuarios, por momentos guardando un orden de llegada para ser recibidos. Allí se dan interacciones entre los sujetos, se intercambia información de manera rápida y al mismo tiempo en 
forma atenta, con lo cual ser conciso resulta de gran importancia: recetas de cocina, aplicación de algún medicamento, y los típicos actos de habla constituyentes de reciprocidades, es decir saludos y otras fórmulas rituales de reconocimiento más general (Austin, 1982; Goffman, 1970).

Allí tuvo lugar un acontecimiento que consideramos de gran significación para comprender el tipo de procesos mediacionales que se llevan a cabo en las policlínicas de este tipo. Una tarde, durante el turno de medicina general y otras especialidades, la sala de espera se encontraba en pleno dinamismo. Una abuela afro-descendiente no paraba de tejer muy concentrada, mientras su muchachito y otro amigo jugaban fuera, varios grupos de mujeres dialogaban sobre patologías, vida cotidiana, relaciones familiares y sociales, una paciente esperaba fuera fumando un cigarrillo, y el sol penetraba profusa-mente con su bajada camino al horizonte. Fruto de la creciente ampliación de los servi-cios prestados por la policlínica, en medio del proceso de crecimiento que ya hemos descrito y analizado, se desenvuelven acontecimientos que si bien no son del todo ex-traños tampoco dejan de ser novedosos.

De pronto, sentados en nuestra posición en el banco largo del fondo, se sienten las voces de la auxiliar y una de las usuarias (quien se encontraba con su hija y nieta de pocos meses de vida). La primera dice: "dale, poné la pierna flojita, no te muevas", lo que logra escucharse junto a risillas entrecortadas, algo nerviosas pero confiadas, de las demás involucradas. Todos quienes estamos en la sala de espera nos mostramos atentos a ello, mirando hacia la fuente de emisión de aquellos sonidos e inclinando hacia allí nuestros cuerpos, una camilla a un lado del espacio intersticial. Se trataba de una extrac-ción de sangre, servicio nuevo en la policlínica. Ya una de las médicas, quien ordenó dicho acto, lo había manifestado en voz alta cruzando la sala. Su hija y una joven cerca-na, y alguien más se encontraban de ese lado, junto a ella, acompañando tanto a la pa-ciente como a la funcionaria.

Cuando terminó la extracción se multiplicaron las risas, sonaron palabras en por-tuñol, el dialecto portugués del Uruguay (pues la adolescente madre era oriunda de la frontera con Brasil, le cantaba a su bebé en portugués y la auxiliar anteriormente le había consultado delante de todos los presentes sobre sus sentimientos acerca de Rivera, su lugar de procedencia). "Pasó lo peor" se escuchó; la sensación de tranquilidad posterior al acontecimiento, puso al descubierto el tipo de experiencias que ahora pueden susci-tarse en la policlínica, dado el crecimiento y cambio cualitativo de su condición intermedia entre lo local y lo zonal.

\section{E1 estilo de comunicación adolescente: construcción de identidades}

Las adolescentes en este contexto ocupan un rol particular y evidencian modali-dades comunicacionales específicas. Si la dinámica lógica y compositiva de grupos en permanente movimiento de armado y desarmado, a veces más acelerado otras muy len-tamente, opera por lo general en los espacios de espera de las policlínicas de este tipo, las usuarias jóvenes no solo no constituyen una excepción, sino que por el contrario son un perfil de usuario fuertemente socializado de tal forma. Los pequeños grupos de jóvenes y adolescentes que comparten sus días y turnos de consulta son un fenómeno carac-terístico.

Sus diálogos son uno de los elementos constituyentes del paisaje comunicacional: sobre amigos, episodios con teléfonos móviles, compras, especialmente de ropa. En toda mediación, en la articulación de 
diversos medios y soportes en usos específicos, maneras de hacer la comunicación y con ella de producir, distribuir y recibir información, existen estilos, formas singulares y singularizantes, y ello es finalmente lo que nos coloca en la dimensión de la producción de subjetividad.

El estilo de comunicación adolescente, podríamos decir, en estos contextos investigados, se caracteriza por el uso de la jerga, el argot, la lengua propia de su dimensión intersubjetiva. Se encuentran ante otras mujeres, en gran medida de la edad de sus adultas, pero interactúan similarmente a como lo hacen entre sí en otros espacios de socialización de lo cotidiano, especialmente los centros educativos donde pueden llegar inclusive a ser compañeras de aula, así como en otros espacios públicos en su localidad.

Reafirmando nuestra idea de que lo local se hace sentir fuertemente en estas instancias a pesar de estar institucionalizadas por fuerzas más abstractas como las de un sistema estatal sanitario, el relacionamiento adolescente también se ve afectado por esta condición, y quizás este hecho es de los indicadores más evidentes de lo que sucede a nivel general. Palabras para las que se considera imprudente su uso en los denominados "espacios públicos", las llamadas "malas-palabras" especialmente desde las comunida-des de habla, esferas de uso y géneros discursivos (Bajtín, 1982) claramente marcados por las diferencias etarias, atravesados a su vez por las diferencias de sectores socioeco-nómicos al estilo de los "sociolectos" (Coseriu, 1981), son proferidas en discursos a un volumen más alto por muchos momentos de la media del lugar.

De esa forma, considerando que no hace falta reservar las palabras para los oídos de los integrantes del pequeño grupo $-y$ especialmente si tomamos en cuenta la presencia del etnógrafo, adulto y varón- se suceden las cuestiones relativas a la moda, a cuáles y cómo usar "calzones" (ropa interior), cómo relacionarse con otras jóvenes, aquellas que "andan mostrado las tetas". "Chusmeando" sin cesar, regando el habla de risas y carcajadas, se intercambia información sobre las relaciones entre sí y otros de su misma condición (quién, por ejemplo "se banca las cargadas", es decir, se adapta a los dichos descalificatorios de los demás), en un proceso que podemos reconocer como de produc-ción de identidad, asociado al tipo de adolescencia en una configuración concreta. Los contenidos más relevantes, por tanto, pasan por esta construcción identitaria, en el sentido de los vínculos con los pares, según el género principalmente, así como la expresividad de todo ello, en especial en lo relativo a la vestimenta y la presentación de la " $f a$ chada" en los espacios de socialización (Goffman, 1971), y el uso del propio lenguaje al que hacemos nosotros a su vez referencia, la performance lingüística, evidenciando la función meta que ya sabemos la caracteriza y que junto a la referencial constituyen la reflexividad presente en lo cotidiano (Lefebvre, 1967: 174).

Sería irnos de la temática principal de la investigación general en la que se ins-cribe esta indagación etnográfica, querer analizar en particular este discurso, por lo cual no podemos afirmar cuales son los pesos relativos y la composición del conjunto a partir del estudio pormenorizado de los enunciados y las formas discursivas en general pre-sentes, como ser la de las adultas de la misma localidad, las profesionales del campo sanitario, en su diversidad de especialidades, diferencias etarias, etc.

Pero parece evidente que existe una cercanía mayor entre las adolescentes y las otras mujeres del mismo contexto cotidiano 
que con las profesionales del sistema, sean más jóvenes o más adultas. Es así que es corriente que alguna adulta se inmiscuya en las conversaciones de estas muchachas, como sucede en la generalidad de esta dinámica vincular.

En una oportunidad, frente a diálogos acerca de lo caro que resulta la compra de calzado deportivo, las "bases" tan valoradas en los modelos juveniles de consumo asociados a la música tropical, la llamada identidad plancha y sus extensiones (Venturini, 2010), una adulta exclamó: “sí, y después te los roban".

Las condiciones de existencia compartidas, relaciones previas y todo el entrama-do vecinal entre quienes son del mismo territorio o de algunos contiguos y ya se reco-nocen en espacio-tiempos como este, se afianzan en una feminidad compartida desde la maternidad, ya desde el embarazo.

Las adolescentes en estado de gravidez integran estos grupos con sus amigas y conocidas, así como llegan junto a sus referentes adultas. También están las jóvenes madres, con sus bebés, solas o más en general acompañadas a su vez por sus madres. Entre ellas se relacionan, pasando de grupo en grupo momentáneamente y volviendo con sus pequenos.

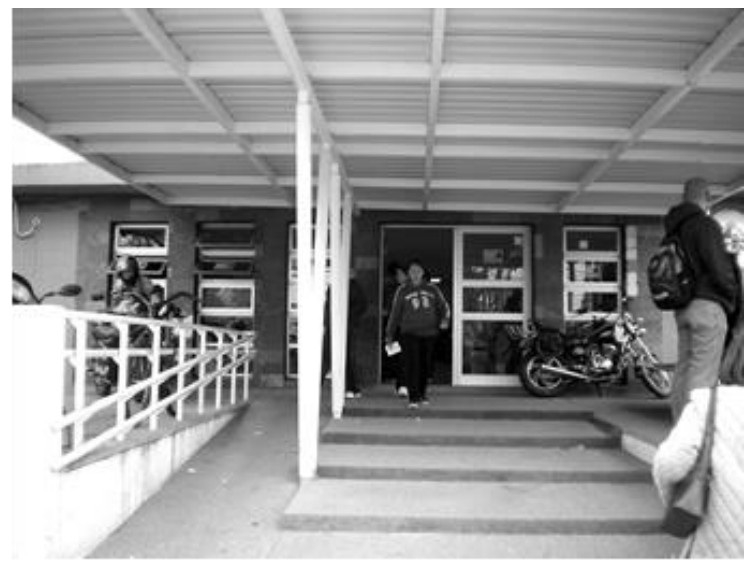

Figura 8: Centro de salud Jardines del Hipódromo

\section{Consideraciones finales}

Através de este tipo de indagaciones pretendemos dejar en evidencia el poten-cial y las capacidades que existen en los propios territorios donde actúan determinado tipo de políticas sociales, como en este caso, orientadas desde la promoción de la salud en jóvenes y adolescentes del oeste del Departamento de Montevideo en Uruguay. El abordaje etnográfico permite la realización de este tipo de cartografías que son de gran utilidad para establecer un diálogo con lo existente, estos espaciotiempos de interac-ción. Hemos intentado profundizar en todo ello a partir de lo que la comunicación y la información conforman en tales entidades.

Los procesos de transversalización que desterritorializan a los sujetos y los vuel-ven a territorializar permanentemente, encuentros-desencuentros donde son posibles de forma relativa estas vinculaciones inter y trans-subjetivas. Hemos visto, por tanto, cuáles eran los tipos de mediaciones características, buscando comprender cómo se sostienen los medios y los tipos de mensajes, las expresiones y los contenidos, en sus relaciones inextricables, su composición particular. Además, hemos planteado cómo la espacialidad y temporalidad que se configura en una "espera" no es algo neutro ni estable, dado que existen diferentes formas de estar presente, agenciamientos que conllevan modalidades de participación, maneras de experimentar una situación.

Un segundo aspecto importante es la experiencia del dinamismo de los contextos de investigación, dándose la reproducción de cierto tipo de condiciones al mismo tiempo que modificaciones sustanciales. En este caso en particular, la policlínica local, todo COVIPRO y la micro-zona donde se inscribe, se ven fuertemente alteradas por los nue-vos emprendimientos territoriales $y$ 
urbanísticos. Al mismo tiempo, aumenta la presencia de usuarios de localidades cercanas $\mathrm{y}$ se suman nuevos servicios por la conjugación de varios factores médicos, de gestión de las políticas sociales, de las comunidades asis-tidas, lo que se traduce en la forma en que se intensifica la atención sanitaria y conlleva nuevas experiencias posibles, acontecimientos donde se ponen en juego otras dimensio-nes existenciales (como la suscitada por la extracción de sangre).

Finalmente, resaltamos una vez más el interés por estos universos o campos de experiencia, tan vivos y ricos en acontecimientos, siempre en conexión a otros gracias a las fuerzas que los atraviesan y a su vez les dan consistencia, lo comunicacional e in-formacional que pone en juego una multiplicidad de mundos.

\section{Bibliografía}

1. ÁLVAREZ PEDROSIAN, Eduardo, 2009a, "Casavalle: una zona, un barrio, un lugar. Periferia urbana y fragmentación de la subjetividad", en Actas electrónicas de las VIII Jornadas de Investigación de la Facultad de Ciencias Sociales: El futuro del país en debate. Montevideo, Facultad de Ciencias Sociales-UdelaR.

2. —, 2009b, Los estrategas del Maciel. Etnografía de un hospital público. Montevideo, CSIC-UdelaR.

3. - 2010, "Espacios de internación hospitalaria: desafíos para la etnografía de la subjetividad", en RO

4. MERO GORSKI, S. (comp.y ed.) Anuario de Antropología Social y Cultural en Uruguay 2009-10. Mon-tevideo, NordanComunidad - Facultad de Humanidades y Ciencias de la Educación-UdelaR, 123-140.
5. AUSTIN, John Langshaw, 1982, Cómo hacer cosas con palabras: palabras y acciones. Barcelona, Pai-dós.

6. BAJTÍN, Mijaíl, 1982, "El problema de los géneros discursivos", en Estética de la creación verbal. Mé-xico, Siglo XXI, 248293.

7. BENECH, A. SPRECHMANN, Thomas, 1995, "Hospitales: complejidad o anarquía", en Elarqa, 5 (14): Arquitectura y salubridad, buena salud.

8. BOURDIEU, Pierre, (dir.), 2000, La miseria del mundo. Buenos Aires, FCE.

9. CABRERA, Marisol. RICARDO, Lourdes BRESCIANO, Ma. Lourdes, 2009, "La divulgación científica de la salud a través de una experiencia radial en un liceo de Montevideo", en Actas electrónicas del Foro iberoamericano de comunicación y divulgación científica, Campinas, OEIUNICAMP, en «http://oei.es/forocampinas /PDF_ACTAS/COMUNICACIONES/gru po3/033.pdf〉 (acceso en 20/06/2012).

10. CHATMAN, Elfreda. A., 1999, "A theory of life in the round", en Journal of the American Society for Information Science \& Technology 50 (3): 207-217.

11. COSERIU, Eugen, 1981, Lecciones de lingüística general. Madrid, Gredos.CPP. s/d, "Proyectos Históri-cos. Programa Cooperativa de Viviendas El Progreso (COVIPRO), Ruta 8, Brig. General Lavalleja, Km. 18.500, Padrón 60008, Montevideo", en 〈http://cpp.org.uy/ proyectoshistoricos/territorial/C214.htm> (acceso en 15/04/2012)

12. DEL CASTILLO, Alina. MELLO, Luciana. PIAZZA, Norma. LARROCA, Jorge, 2012, "Interdisciplina en campo. Espacio de formación integral: hábitat y territorio", en Revista Digital Universitaria, 13 (5), en 〈http://www.revista.unam. 
$\mathrm{mx} /$ vol.13/num5/art51/\#up〉 (acceso en 18/05/2012).

13. DELEUZE, Gilles. GUATTARI, Felix, 1997, Mil mesetas. Capitalismo y esquizofrenia II. Valencia, Pre-textos.

14. GOFFMAN, Ervin, 1970, Ritual de la interacción. Buenos Aires, Tiempo contemporáneo.

15. GUATTARI, Felix, 1996, Caosmosis. Buenos Aires, Manantial._, 1971, La presentación de la persona en la vida cotidiana. Buenos Aires, Amorrortu.

16. HALL, Edward T., 1994, La dimensión oculta. México, Siglo XXI.

17. LEFEBVRE, Henri, 1967, Lenguaje y sociedad. Buenos Aires, Proteo.

18. LEVI-STRAUSS, Claude, 1970, Tristes trópicos. Buenos Aires, EUDEBA.

19. LOLAS STEPKE, Fernando, 1997, Más allá del cuerpo. La construcción narrativa de la salud. Santiago de Chile, Andrés Bello.

20. LUDUEÑA, Alicia del Carmen, OLSON, Joanne, PASCO, Alberta, 2005, "Promoción de la salud y cali-dad de vida entre madres de preadolescentes. Una etnografía enfocada", en Revista LatinoAmericana de Enfermagem, 13, 1127-1134, en «www.eerp.usp.br/rlae) (acceso en 15/04/2012).

21. MATTELART, Armand. MATTELART, Michèle, 1997, Historia de las teorías de la comunicación. Barcelona, Paidós.

22. MARTÍN-BARBERO, Jesús, 2003, De los medios a las mediaciones. Bogotá, Andrés Bello - Gustavo Gili.

23. ONG, Walter, 1996, Oralidad y escritura. Tecnologías de la palabra. México, FCE.

24. PEÑARANDA-CORREA, Fernando, 2011, "Las fuerzas sociales y el escenario comunicativo en la edu-cación para la salud: el caso del programa de crecimiento y desarrollo", en Ciência \& Saúde Coletiva, 16 (10), en http://dx.doi.org/10.1590/S141381232011001100007) (acceso en 18/07/2012).

25. SAVOLAINEN, Reijo, 2008, Everyday information practices: a social phenomenological perspective. Lanham, Scarecrow Press.

26. SIMMEL, Georg, 2005, "La metrópolis y la vida mental", en Bifurcaciones. Revista de estudios cultura-les urbanos, 4, en «www.bifurcaciones.cl) (acceso en 12/03/2012).

27. VENTURINI, Joaquín, 2010, "Etnografía de una banda de cumbia villera. Hacia una comprensión de la identidad plancha desde las industrias culturales", en Actas electrónicas de las IX Jornadas de Investiga-ción en Ciencias Sociales. Montevideo, Facultad de Ciencias SocialesUdelaR, en http://www.fcs.edu.uy/ archivos/Venturini.pdf) (acceso en 7/04/2012).

28. VIDART, Daniel, 1998, La trama de la identidad nacional. Tomo II: El diálogo ciudad-campo. Monte-video, EBO.

Otras fuentes

29. Administración de los Servicios de Salud del Estado (ASSE), 2012, Portal de la Red de Atención de Pri-mer Nivel (RAP), en 〈http://www.asse.com.uy/categoria_209_1_ 1.html (acceso en 10/06/2012). Instituto Nacional de Estadística (INE), 2005, Unidad de Cartografía, Mapa Sección Censal 99, en 〈http://www.ine.gub.uy/mapas/01\%20Por $\% 20$ Seccion/a0v_99-1.pdf (acceso en 20/06/2012). 